\title{
CNS demyelination due to hypocupremia in Wilson's disease from overzealous treatment
}

\author{
Sunil K. Narayan, Nandigam Kaveer \\ Department of Neurology, Jawaharlal Institute of Postgraduate Medical Education and Research, Pondicherry - 6, India
}

Untreated Wilson's disease in advanced stage is associated with high neurological morbidity, and effective treatment with chelating agents and zinc salts has shown to prevent the development of or reverse neurological symptoms. ${ }^{[1]}$ However an overzealous treatment with zinc over a prolonged period can have its own dangers.

A 13-year-old boy presented with symptoms of difficulty in using hands, difficulty ambulating, and slurred speech for six months duration. He was diagnosed to have Wilson's disease at the age of nine years based on the presence of Corneal KF ring and serum ceruloplasmin value of $27.5 \mathrm{mg} / \mathrm{dl}$ (Normal range: 20-50 mg/dl). His CT brain had then showed bilateral caudate nucleus atrophy and normal cerebral hemispheres. He was then started on treatment with penicillamine $750 \mathrm{mg} /$ day and Zinc Sulphate Monohydrate $280 \mathrm{mg}$ /day. His mother reported good compliance with the prescribed medication for the past four years. On examination, KF ring was still present on the superior and inferior margins of both corneae. He had spastic dysarthria, a speech characterized by echolalia and occasional outbursts of panic. Neurological examination also showed saccadic dysmetria, decreased vibratory and joint position sense in the feet, brisk tendon reflexes, extensor plantar responses, marked truncal ataxia, dystonic posturing of both hands and feet and cogwheel rigidity at the wrists. Serum ceruloplasmin $2.34 \mathrm{mg} / \mathrm{dl}$, serum copper 16 $\mu \mathrm{g} / \mathrm{dl}$ and serum Zine $155 \mu \mathrm{g} / \mathrm{dl}$ and a normocytic normochromic anemia with anisopoikilocytosis. CT brain showed diffuse hypodensity involving white matter tracts bilaterally, attenuation in the range of $15-18 \mathrm{Hu}$, consistent with demyelination, predominantly in the frontal, parietal, and temporal regions, sparing the posterior. Prominence of the bilateral lateral ventricles and the third ventricle was seen along with mild cerebral and basal ganglia atrophy region [Figures 1 and 2]. Brain stem and cerebellum appeared normal.

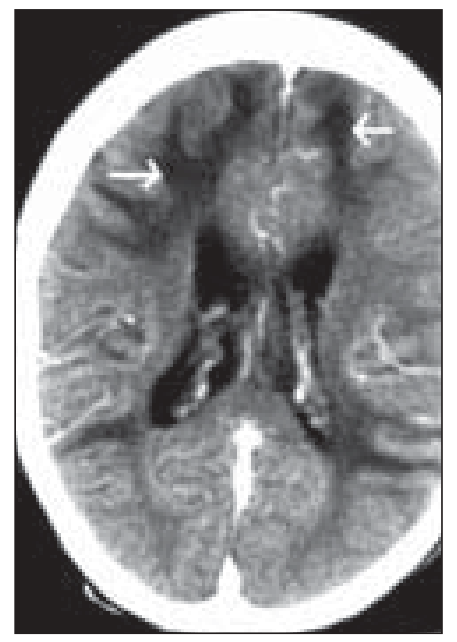

Figure 1: CT scan Brain showing prominent symmetrical demyelination in subcortical white mater of frontal lobes

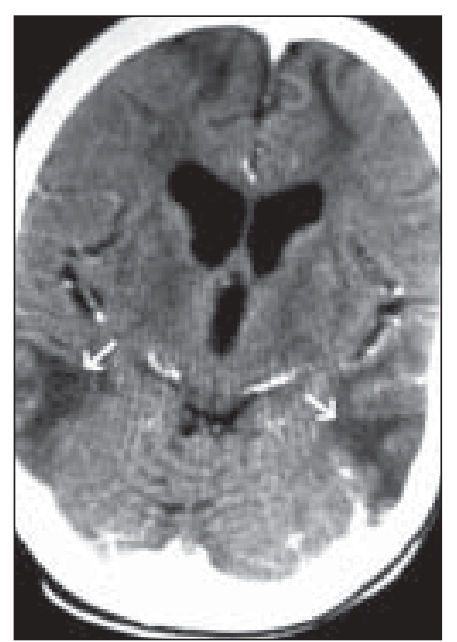

Figure 2: Similar demyelination symmetrically in parieto-temporal lobes 


\section{Discussion}

At the initial diagnosis of Wilson's disease in this patient, no CNS symptoms were reported and CT brain was remarkable only for bilateral caudate nucleus atrophy and his serum ceruloplasmin then was in the low normal range, i.e. $27.5 \mathrm{mg} / \mathrm{dl}$. But after four years of treatment with $750 \mathrm{mg} / \mathrm{d}$ penicillamine, which is equivalent to the adult maintenance dose, and $280 \mathrm{mg} /$ $\mathrm{d}$ of zine, which is nearly double the adult maintenance dose, the serum ceruloplasmin was virtually undetectable and serum copper $(16 \mu \mathrm{g} / \mathrm{dl})$ was much lower than the normal range of 80 to 120 $\mu \mathrm{g} / \mathrm{dl}$. As reported by Prodan et al. ${ }^{[2]} \mathrm{CNS}$ demyelination seen in this patient is emphatically attributable to the hypocupremia. A possible mechanism for the demyelination could be hypocupremia leading to deficiency of many copper containing metalloenzymes such as superoxide dismutase (SOD), which plays a vital role in protection against oxidative damage..$^{[3]}$ This case exemplifies the importance of frequent monitoring of serum copper and ceruloplasmin levels in Wilson's disease patients undergoing treatment, specially if they are in the pediatric age group, and adjustment of the dosages of the drugs accordingly, to prevent further neurological complications such as CNS demyelination ${ }^{[2]}$ and myeloneuropathy ${ }^{[4]}$ due to hypocupremia.

\section{Acknowledgement}

We acknowledge the Public Health Laboratories, Government of Pondicherry for their assistance on biochemical estimations.

\section{References}

1. Brewer G.J. Neurologically presenting Wilson's disease: epidemiology, pathophysiology and treatment. CNS Drugs 2005;19:185-92.

2. Prodan CI, Holland NR, Wisdom PJ, Burstein SA, Bottomley SS. CNS demyelination associated with copper deficiency and hyperzincemia. Neurology 2002;59:1453-6.

3. Marx J. Mutant enzyme provides new insights into the cause of ALS. Science $1996 ; 271: 446-7$.

4. Rowin .J, Lewis SL. Copper deficiency myeloneuropathy and pancytopenia secondary to overuse of zinc supplementation. J Neurol Neurosurg Psychiatr $2005 ; 76: 750-1$.

Accepted on 19-01-2006 